\title{
Re-sternotomy for aortic valve replacement in octogenarian patients Submission category - Original article
}

\author{
Suvitesh Luthra ${ }^{1}$, Pietro Giorgio Malvindi ${ }^{1}$, Hannah Masraf ${ }^{2}$, Anna Podonyi $^{1}$, Taha \\ Ramadan $^{1}$, and Sunil Ohri ${ }^{1}$ \\ ${ }^{1}$ University Hospital Southampton NHS Foundation Trust \\ ${ }^{2}$ University of Southampton
}

November 19, 2021

\begin{abstract}
Background The aim of this study was to analyse the perioperative results and long term survival of re-sternotomy for surgical aortic valve replacement (SAVR) in octogenarians. Methods This is a retrospective, single centre study (Apr 2000 - Dec 2019). Perioperative data were compared for re-sternotomy with isolated SAVR (Isolated redoSAVR) and re-sternotomy with SAVR and concomitant cardiac procedure (Associated redoSAVR). Regression analyses were performed to identify predictors of in patient mortality. Hazard ratios were calculated, and Kaplan Meier survival curves were compared for groups. Results There were 163 patients (Isolated redoSAVR; 69, Associated redoSAVR; 94). Mean age was $83 \pm 3$ years and mean logEuroSCORE was $21 \pm 12$. Follow up was $4.2 \pm 3.5$ years. Inpatient mortality was $4.9 \%$ ( $1.4 \%$ versus $7.4 \%$ for Isolated redoSAVR and Associated redoSAVR respectively, $\mathrm{p}=0.08)$. TIA/stroke rate was $8 \%(9 \%$ versus $7 \%$ for Isolated redoSAVR and Associated redoSAVR respectively, $\mathrm{p}=0.78)$. COPD was a predictor of inpatient mortality (OR; 8.86, 95\%CI; 1.19, 66.11, $\mathrm{p}=0.03)$. Survival was $88.7 \%$ at 1 year, $86.4 \%$ at 2 years, $70.1 \%$ at 5 years, $49.5 \%$ at 7 years and $26.3 \%$ at 10 years. There was no survival difference between Isolated redoSAVR and Associated redoSAVR (logrank $\mathrm{p}=0.36$, Wilcoxon $\mathrm{p}=0.84$ ). Significant predictors of adverse long term survival were COPD, postoperative TIA/stroke and length of stay. Survival is lower than age and gender matched first time SAVR and general population of UK. Conclusions RedoSAVR in octogenarians is associated with acceptable but significant morbidity and mortality. Shared decision making should consider emerging transcatheter therapies as viable options in selected patients.
\end{abstract}

\section{Hosted file}

resternotomy in octognarians JCS.docx available at https://authorea.com/users/350217/ articles/546137-re-sternotomy-for-aortic-valve-replacement-in-octogenarian-patientssubmission-category-original-article 